\title{
Abordaje de los sintomas neurológicos desde la perspectiva de los cuidados paliativos
}

\section{Neurological symptoms approach from the perspective of palliative care}

\author{
Luz-Eugenia Ibáñez-Alfonso ${ }^{1,2}$; Julián-Alberto López-Alba ${ }^{1,3}$; María-Angélica Ramírez-Ospina ${ }^{1}$; \\ Martha-Cecilia Escobar-Ruiz'; Miguel-Antonio Sánchez-Cárdenas ${ }^{1,4}$
}

Forma de citar: Ibañez Alfonso LE, López Alba JA, Ramírez Ospina MA, Escobar Ruiz MC, Sánchez Cárdenas MA. Abordaje de los síntomas neurológicos desde la perspectiva de los cuidados paliativos. Rev Univ Ind Santander Salud. 2018; 50(3): 233-245. doi: http://dx.doi.org/10.18273/revsal.v50n3-2018008 @c) (i)

\section{Resumen}

Objetivo: Abordar los síntomas neurológicos desde la perspectiva de los cuidados paliativos a través de la revisión de literatura científica disponible teniendo en cuenta los componentes teóricos del Modelo Revisado del Manejo de Síntomas de Marylin J. Dodd. Metodología: Siguiendo los pasos propuestos por la declaración Prisma, se realizó revisión sistemática de las publicaciones realizadas entre el año 2002 y 2017 de artículos relacionados que abordaban los síntomas neurológicos desde la perspectiva del cuidado paliativo aplicando los componentes del Modelo Revisado del Manejo de Síntomas de Marylin J. Dodd, los cuales incluyen la experiencia del síntoma, los componentes de las estrategias para el manejo de síntomas y el resultado en términos del estado y el impacto del síntoma. Resultados: De los 60 articulos identificados inicalmente, 50 cumplieron con los criterios de selección y se clasificaron según las categorias de la Teoría Manejo de síntomas. Conclusiones: El aporte multidisciplinario optimiza el control de síntomas y la calidad de vida, pero son las enfermeras las responsables de detectar las respuestas humanas y distinguir entre el dolor y la agitación, equilibrar los riesgos de sub y sobremedicación y la posibilidad de acelerar la muerte. Se necesitan enfoques innovadores para alinear los comportamientos profesionales de la salud con el mejor cuidado donde se incluya una atención integral que mejore significativamente la atención al paciente.

Palabras clave: Síntomas neurológicos; Cuidado paliativo; Enfermería; Enfermo termial; Delirio.

\begin{abstract}
Objective: To address neurological symptoms from the perspective of palliative care through the review of available scientific literature taking into account the theoretical components of the Revised Symptom Management Model of Marylin J. Dodd. Methodology: Following the steps proposed by the PRISMA statement, a systematic review was made of the publications made between 2002 and 2017, of related articles that addressed neurological symptoms from the perspective of palliative care applying any of the components of the Revised Symptom Management Model of

1. Universidad El Bosque. Bogotá D.C. Colombia.

2. Universidad Industrial de Santander. Bucaramanga, Colombia.

3. Universidad de los Llanos. Villavicencio, Colombia

4. Observatorio de Cuidados Paliativos de Colombia. Bogotá D.C. Colombia

Correspondencia: Luz Eugenia Ibáñez Alfonso. Dirección: Carrera 26 35-31, Floridablanca Teléfono: +57 300 5571819. Correo electrónico: libanez@saber.uis.edu.co
\end{abstract}


Marylin J. Dodd, which include the experience of the symptom, the components of symptoms management strategies and the result in terms of the state and impact of the symptom. Results: according to the search limits defined within the inclusion criteria, 51 articles were selected to be analyzed from the perspective of palliative care applying the components of the Revised Symptom Management Model. It is evident that there is a significant amount of research that addresses delirium as a neurological symptom. Discussion: The multidisciplinary contribution often optimizes the control of neurological symptoms and the quality of life of patients and their families. In the same way, it is important to learn the use of scales and the early recognition of the impact of the symptom on the patient and his family to significantly improve the adaptation process. Conclusions: Although there are not sufficient innovative approaches to improve the management of symptoms as a whole and not as individual manifestations, education and training in the use of non-pharmacological interventions help to provide quality palliative care that complements to the traditional managements.

Keywords: Neurologic manifestations; Palliative care; Nursing; Terminally ill; Delirium.

\section{Introducción}

Los cuidados paliativos se han convertido en un componente esencial de situaciones terminales en donde se busca maximizar la calidad de vida, lograr un adecuado control del dolor y los síntomas. La gestión se centra en identificar y tratar las causas reversibles, respetando los valores y las metas del paciente ${ }^{1}$. Los objetivos de este abordaje deben establecerse de forma individual de acuerdo con la autonomía del paciente, es imperativo planificarlos anticipadamente en escenarios críticos específicos en donde es útil tratar a los pacientes teniendo en cuenta sus deseos y valores ${ }^{2,3}$.

Si bien es cierto, los cuidados paliativos son estándar para pacientes en condiciones crónicas y/o limitantes de $v i d a^{4}$, para lograr el bienestar se requiere conocer los síntomas más comunes, los medicamentos para el alivio, las vías de administración, la efectividad de los mismos y las situaciones en que se producen incompatibilidades, interacciones y efectos adversos ${ }^{5}$.

Un enfoque paliativo centrado en la comodidad, la calidad de vida y el apoyo familiar puede tener beneficios para los pacientes, las familias y el sistema de salud, ya que mejoran la relación costo-efectividad de la atención, disminuyen las hospitalizaciones no deseadas y la atención agresiva al final de la vida ${ }^{6}$. Esta etapa en la cual se presentan distintas crisis, plantea una serie de desafíos excepcionales para los pacientes, familiares y los profesionales de la salud ${ }^{2}$, pues son numerosos los factores que impiden o demoran la comunicación efectiva y oportuna durante el cuidado 7 . Es necesario considerar la complejidad en los patrones de alivio de los síntomas para que los grupos de personas comprendan y evalúen las mejoras en el padecimiento de los síntomas como un indicador de la calidad de la atención al final de la vida ${ }^{6}$.
Desde la perspectiva de los cuidados paliativos es importante considerar a las personas que cursan con patologías distintas a la oncológica. Esto se hace evidente en enfermedades neurológicas que, a pesar de tener un pronóstico distinto, muchas de ellas comparten atributos como el hecho de ser crónicas, incurables y limitantes en lo que se refiere a la autonomía del paciente $^{8}$. Para el abordaje y la planificación del cuidado paliativo, en estos casos que involucran afectación del estado neurológico, ya sea desde el componente sensitivo o motor, es necesario considerar la complejidad de la enfermedad, el curso clínico, el pronóstico, las consecuencias sobre la independencia y la variabilidad de los síntomas 9 .

La revisión de literatura ha evidenciado que las enfermedades neurológicas en Estados Unidos y en otros países afectan a millones de personas ${ }^{5}$. La incidencia de síntomas neurológicos y secuelas resultantes de este tipo de padecimientos, en su mayoría crónicos, ha venido aumentando ${ }^{4}$. Estos tipos de reportes en Latinoamérica y Colombia son escasos y algunos de ellos mencionan la dificultad para establecer estas cifras por el gran subregistro de las patologías $2,3,6,7,10-12$.

De acuerdo al estudio realizado por la Fuerza de Tarea de la Asociación Europea de Cuidado Paliativo en colaboración con el Panel Científico de Cuidado Paliativo en Neurología ${ }^{13}$, cada vez hay más pruebas de que los cuidados paliativos y un enfoque multidisciplinario, integrados tempranamente en la atención mejoran los síntomas y la calidad de vida de los pacientes y sus familias, siendo necesario establecer una comunicación fluida con ellos, la planificación de atención avanzada, el manejo de síntomas, el cuidado al final de la vida, el apoyo y capacitación del cuidador y la educación para todos los profesionales involucrados en la atención. 
La mayoría de la sintomatología neurológica crónica es incurable y el tratamiento se basa principalmente en el manejo para mejorar la calidad de vida ${ }^{14}$. Padecimientos neurológicos que requieren cuidados paliativos como la Esclerosis Lateral Amiotrófica, las enfermedades neuromusculares, la Miastenia Gravis, la Distrofia muscular, la Demencia, los desórdenes del movimiento, la Enfermedad de Parkinson, los desórdenes autonómicos en la Atrofia Sistémica Múltiple, la Parálisis supranuclear Progresiva, la Enfermedad de Huntington, la Esclerosis Múltiple, los tumores cerebrales, las enfermedades cerebrovasculares y la lesión cerebral traumática producen una serie de alteraciones motoras, neurocomunicativas, cognoscitivas y emocionales que interfieren en la funcionalidad e independencia del paciente ${ }^{15}$.

De acuerdo al texto de Oxford de Enfermería Paliativa ${ }^{16}$, los síntomas neurológicos son numerosos e incluyen movimientos anormales (convulsiones, mioclonías, ataxia, fasciculaciones, disquinesia, tremor, nistagmus, etc), distintos tipos de cefaleas, espasticidad, delirio, agitación, confusión, afasia, entre otros. El presente estudio tiene como objetivo abordar los síntomas neurológicos desde la perspectiva de los cuidados paliativos, a través de la revisión de literatura científica disponible teniendo en cuenta los componentes teóricos del Modelo Revisado del Manejo de Síntomas de Dodd, et al., propuesto en el año $2001^{17}$.

\section{Métodología}

La revisión de literatura científica sobre el tema desarrollado, utilizó la estrategia $\mathrm{PICO}^{18}$, (Población, Intervención, Comparación, Resultado), determinando como elementos conceptuales los siguientes: P, Pacientes adultos con enfermedad en etapa terminal. I, Cuidados Paliativos, Manejo de síntomas neurológicos, $\mathrm{C}$, no aplica. O, control de signos neurológicos.

Pregunta clave: ¿Cuáles son las estrategias de cuidados paliativos para el manejo de síntomas neurológicos en los pacientes adultos en etapa terminal?

Metodología de selección de artículos:

Para cada búsqueda, a partir del conjunto de referencias encontradas en cada base de datos, se realizó la selección de los potenciales artículos elegibles de acuerdo con los límites de búsqueda establecidos mediante el proceso de selección de la revisión por títulos, luego filtrado por resúmenes, y finalmente selección por lectura de texto completo.

Siguiendo la declaración PRISMA ${ }^{18}$ (Preferred Reporting Items for Systematic Reviews and MetaAnalyses) $^{19}$, se realizó una revisión sistemática aplicando cada uno de los pasos propuestos: identificación, cribado, idoneidad e inclusión.

Durante el periodo comprendido entre los meses de marzo y mayo de 2018, se realizó una búsqueda en las bases de datos Pubmed, Ebsco Host, Embase, Clinical Key y Proquest, utilizando para ello distintos protocolos con términos normalizados de acuerdo a los Descriptores Mesh: Terminally Ill, palliative care, neurologic manifestations, symptom assessment, Decs: enfermedad terminal, cuidados paliativos, síntomas neurológicos y los Booleanos: AND-OR.

Los criterios de inclusión fueron artículos originales, disponibles en línea, con texto completo, en los idiomas inglés y español, con fecha de publicación superior al año 2004 e inferior al año 2018. Se consideraron aquellos artículos o guías donde se hayan abordado estudios de intervenciones, estrategias, actividades, de cuidado paliativo que muestre efectos de cambio en los pacientes en etapa terminal.

El contenido de los artículos debía incluir información sobre el abordaje de los síntomas neurológicos en cuidado paliativo desde cualquiera de los componentes del Modelo Revisado del Manejo de Síntomas de Marylin J. Dodd, los cuales incluyen la experiencia del síntoma, los componentes de las estrategias para el manejo de síntomas y el resultado en términos del estado del síntoma. Se consideraron los adultos mayores de 60 años en estudios experimentales, casos y controles, cualitativos, de cohorte y revisiones sistemáticas y meta análisis.

Se elegieron todos aquellos artículos que potencien el manejo paliativo de síntomas neurológicos en el adulto mayor con enfermedad en etapa terminal.

Los artículos repetidos se consideraron una única vez, se excluyeron de la selección las tesis doctorales, los artículos de prensa y los artículos de reflexión (Tabla 1).

En la Figura 1 se detalla el proceso por medio de un flujograma. 
Tabla 1. Información cuantitativa del proceso descrito anteriormente.

\begin{tabular}{ccccc}
\hline \multicolumn{2}{c}{ Búsqueda Inglés-Español } & \multicolumn{2}{c}{ (Terminally Ill, Palliative Care, Neurologic Manifestations, Symptom Assessment) } \\
\hline Base de Datos & $\begin{array}{c}\text { Resultados } \\
\text { Obtenidos }\end{array}$ & Títulos Seleccionados & Resúmenes Seleccionados & Textos Completos Seleccionados \\
\hline PubMed & 50 & 40 & 20 & 8 \\
Ebsco Host & 40 & 16 & 4 & 2 \\
Embase & 37 & 23 & 7 & 2 \\
Clinical Key & 25 & 20 & 2 & 6 \\
ProQuest & 43 & 15 & 51 & 1 \\
Total & 205 & 115 & & 19 \\
\hline
\end{tabular}

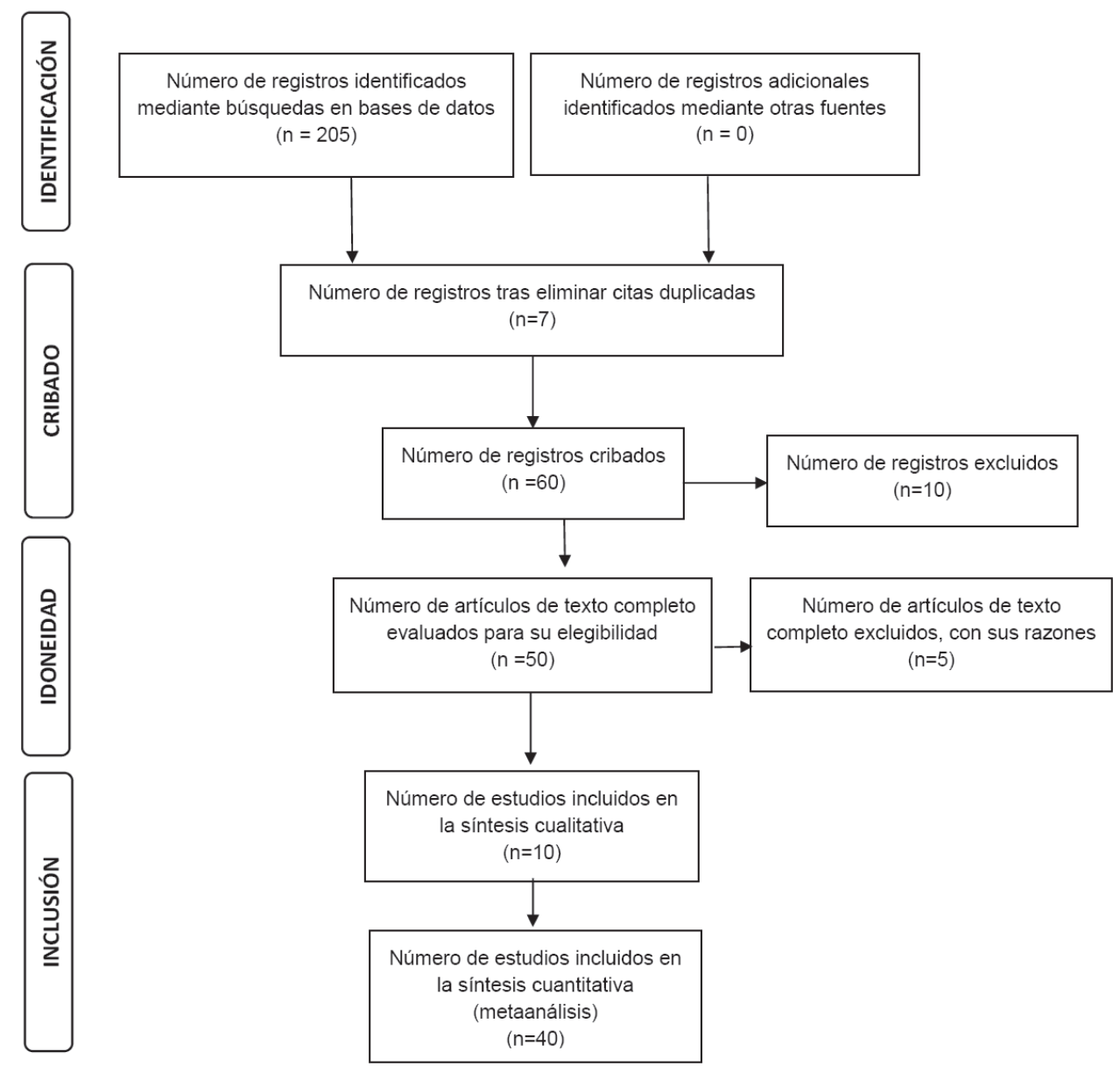

Figura 1. Diagrama de flujo de identificación, selección e inclusión de los estudios ${ }^{19}$.

La revisión de los artículos clasificados según la Teoría del Manejo de Síntomas ${ }^{17}$. Siguió las categorías conceptuales y se analizaron 21 artículos clasificados en la categoría conceptual Experiencia del Síntoma en cuanto a percepción del síntoma, evaluación del síntoma y respuesta al síntoma. Se analizaron 15 artículos clasificados en la categoría conceptual Estrategia Manejo del Síntoma y ocho en referencia a Resultado y Estado del Síntoma.

\section{Resultados}

\section{El Modelo Revisado del Manejo de Síntomas}

La Facultad de Enfermería de la Universidad de California en San Francisco (UCSF) introdujo por primera vez el Modelo de Manejo de Síntomas en 1994 utilizando constructos teóricos de Modelo de Autocuidado de Dorotea Orem y el mismo Modelo 
de Síntomas de Sorofman, sirviendo como marco de referencia para que docentes y estudiantes en la práctica clínica mejoraran el abordaje de los síntomas desde su experiencia, las estrategias de manejo y el resultado (status) de los mismos ${ }^{20}$.

Sin embargo, tras concluir que ninguno de estos tres componentes explicaba adecuadamente el rol, la percepción y las consecuencias deseadas, el modelo fue revisado en el año 2001 por Dodd, et al. ${ }^{17}$ y se incluyeron nuevas etiquetas conceptuales como los dominios de: a) Persona, que incluye las características demográficas, sociológicos, psicológicas, fisiológicas y del desarrollo, b) Salud y enfermedad, que hace referencia a los factores de riesgo, el estado de salud y la presencia de enfermedad o daño y c) Medio ambiente, que abarca los aspectos físicos, sociales y culturales del entorno. Del mismo modo, se plantearon las siguientes premisas:

- El síntoma es una experiencia subjetiva que refleja cambios en el funcionamiento bio-psicosocial, las sensaciones o la cognición de un individuo.

- El estándar de oro para el estudio de los síntomas se basa en la percepción del individuo que experimenta el síntoma y su autoinforme.

- Que el síntoma no tiene que ser experimentado por un individuo para aplicar este modelo de manejo de síntomas. Por ejemplo, un individuo puede estar en riesgo de desarrollar el síntoma por la influencia de factores asociados al trabajo y en este sentido las estrategias de intervención pueden iniciarse de manera preventiva.

- Que los pacientes que no verbalizan (bebés, personas afásicas después del accidente cerebrovascular) pueden experimentar síntomas y se supone que la interpretación por parte del padre o cuidador es precisa para los propósitos de intervenir.

- Que todos los síntomas problemáticos deben ser gestionados.

- Que una estrategia de gestión puede estar dirigida a la persona, un grupo, una familia o el entorno de trabajo.

- Que el manejo de los síntomas es un proceso dinámico; es decir, se modifica por los resultados individuales y las influencias de los dominios de enfermería de la persona, la salud / enfermedad o el medio ambiente.

El Modelo definió tres conceptos mayores ${ }^{17}$ como componentes teóricos, los cuales serán utilizados en el presente estudio para el análisis de la evidencia científica disponible:
- El primer componente de la experiencia del síntoma, el más detallado del modelo, se describe como el comienzo del proceso de manejo de síntomas. Esta dimensión es de gran importancia para el abordaje de los síntomas neurológicos desde el cuidado paliativo debido a que considera la percepción, evaluación y respuesta del individuo a los síntomas y la forma que estos se relacionan con las otras dimensiones.

- El segundo componente del modelo comprende los aspectos individuales de las estrategias de manejo de síntomas. Para el caso de los síntomas neurológicos, es fundamental tener claridad sobre las estrategias farmacológicas y no farmacológicas que se utilizan con el fin de evitar, retrasar o paliar la experiencia del síntoma y mejorar la calidad de vida de individuos, familias y comunidades.

- El tercer componente, es el de resultados del síntoma. Desde la perspectiva de cuidado paliativo, el abordaje de los síntomas neurológicos debe considerar los efectos y consecuencias de estos sobre el estado funcional y emocional del paciente, el autocuidado, los costos, el impacto en la calidad de vida, la morbilidad y la comorbilidad y la mortalidad.

Análisis de resultados a partir de los conceptos teóricos mayores del Modelo Revisado del Manejo de Síntomas

\section{Experiencia del síntoma (percepción, evaluación y respuesta):}

Las alteraciones del sueño se han reconocido por distintos autores ${ }^{21-23}$,como manifestaciones comunes en individuos que padecen enfermedad terminal ${ }^{24,25}$, en pacientes con enfermedad oncológica, la calidad de vida es uno de los mejores predictores de la calidad del sueño ${ }^{22}$. Síntomas como el insomnio y la somnolencia se agudizan y prevalecen más en la última semana de $v \operatorname{da}^{23}$ y se reconoce además que la fragmentación del patrón del sueño es un factor contributivo importante en la progresión temporal del delirio ${ }^{21}$.

El delirio, como complicación neuropsiquiátrica considerada grave, debilitante y multifactorial $^{11}$, puede estar relacionado con procesos infecciosos, la progresión de enfermedades de base como la Esclerosis Múltiple $^{12}$, el estado metabólico o la toxicidad de la medicación. Hay tres dimensiones propuestas de delirio que incluyen la circadiana, la cognitiva y la de pensamiento superior ${ }^{26}$. Al final de la vida este síntoma es muy prevalente ${ }^{27}$ y representa un dilema ético en la práctica clínica: si simplemente tratarlo para maximizar 
el alivio de los síntomas, con el efecto secundario resultante en sedación paliativa, o intentar revertir el delirio y asumir el riesgo de prolongar el sufrimiento ${ }^{28}$.

El delirium ocurre comúnmente en el contexto de los cuidados paliativos, causa mayor angustia en pacientes mayores y cuidadores y hace que la interpretación del dolor y otros síntomas sea extremadamente difícil ${ }^{29}$. La terminología ambigua, la falta de uso de instrumentos de evaluación validados y las distintas definiciones y variables en los sistemas de clasificación reconocidos internacionalmente conducen a una amplia prevalencia e incidencia de episodios de delirio. Los episodios suelen ser multifactoriales y en muchos casos pueden augurar un pronóstico precario. A pesar de esto, muchos son a menudo parcialmente reversibles ${ }^{29}$.

Este síntoma debe considerarse regularmente y discutirse con anticipación para diagnosticarlo precozmente y mejorar su tratamiento ${ }^{26}$. En un estudio realizado por Gagnon, et al. ${ }^{30}$, la incidencia de delirio fue del $49.1 \%$ en el grupo de intervención, en comparación con el $43.9 \%$ en el grupo de atención habitual. En estados terminales, un número considerable de familias experimentó altos niveles de angustia emocional y sintió la necesidad de mejorar el servicio especializado de cuidados paliativos debido a los episodios de delirio que presentaban los pacientes ${ }^{31}$.

Si bien existe una cantidad significativa de literatura disponible sobre delirium en cuidado paliativo en los idiomas inglés y español, no sucede lo mismo con otros síntomas. No obstante, durante la presente revisión se encontró que, al igual que el delirio, la confusión como síntoma neurológico es altamente prevalente en pacientes con cáncer de pulmón ${ }^{32}$, lo cual se ha asociado con un mayor riesgo de muerte ${ }^{33}$. Este síntoma en la práctica clínica ha sido considerado como el mismo delirio ${ }^{34,35}$. Por otro lado, la agitación, en las primeras etapas, puede comenzar como actividad motora frecuente, no intencional, alteración en los patrones de reposo o incapacidad para concentrarse o relajarse, pero puede llegar a incluir un mayor número de síntomas motores, en lugar de psicológicos ${ }^{7}$, y es común encontrarla en pacientes en fase terminal ${ }^{36}$.

En el caso de los movimientos musculares anormales, la prevalencia de convulsiones como eventos paroxísticos, se asocia a enfermedades o condiciones que requieren cuidado paliativo como los tumores primitivos cerebrales, las metástasis y los tratamientos neuroquirúrgicos ${ }^{35}$. Algunos de estos episodios se producen por hipertensión endocraneana ${ }^{34}$. Las mioclonías han ganado una mayor conciencia en el entorno de cuidados paliativos, ya que siendo un fenómeno pasajero o permanente, pueden llegar a provocar un aumento del dolor, la fatiga y otros síntomas angustiantes ${ }^{37}$. Son relativamente frecuentes en el paciente moribundo, aunque éste no suele ser consciente de las mismas. Sus causas suelen ser medicamentosas por opiáceos o por los efectos extrapiramidales de otros fármacos (metoclopramida). Para el caso de la espasticidad, la literatura demuestra que en enfermedades como la esclerosis múltiple la prevalencia reportada es del $40 \%$ y su presentación tiende a cronificarse ${ }^{38}$.

En los adultos mayores, se ha documentado la importancia de la evaluación neurológica rutinaria de la memoria y el pensamiento en lugar de atribuirse al envejecimiento ${ }^{39}$. Los objetivos de manejo para los pacientes con deterioro cognitivo se centran en mantener la función y la independencia, apoyar al cuidador y planificar anticipadamente la atención ${ }^{39}$. En el caso de los individuos con discapacidad intelectual, Tuffrey-Wijne ${ }^{40}$, sugiere que los estudios futuros deberán incluir los puntos de vista y las experiencias de las personas con discapacidad intelectual resaltando así la importancia de la subjetividad del mismo en el abordaje y la toma de decisiones.

Se destacan los resultados del estudio realizado por Heckel, et al. ${ }^{41}$, el cual enfatiza en la necesidad de investigación con enfoques de tratamiento apropiados para mejorar las alteraciones del olfato y del gusto, aspecto relevante en el contexto de atención paliativa si se identifican las quejas quimiosensoriales como carga y angustia para el paciente, por que influye negativamente en su calidad de vida. Hasta ahora el cuidado paliativo parece descuidar la importancia subjetiva de los olores y sabores en la percepción de bienestar de los pacientes.

En cuanto a las herramientas de medición, Fischbeck, et $\mathrm{al}^{4}{ }^{42}$, realizaron un estudio en el que se encontró que la ACPDS (Escala de Dificultad Avanzada para los pacientes con cáncer) puede ayudar a identificar las necesidades de intervenciones de cuidados paliativos en individuos que experimentan angustia. La persistencia significativa de este síntoma se debe principalmente al dolor ${ }^{43}$. Adicionalmente, algunos autores como Spoozak, et al..$^{23}$, Covarrubias, et al. ${ }^{25}$ y Cuervo Pinna, et al. ${ }^{44}$, han utilizado la Escala de Evaluación de Síntomas de Edmonton (ESAS) en pacientes con necesidades de cuidado paliativo y el instrumento Palliative Performance Scale (PPS), instrumentos 
importantes en la estimación de la supervivencia de los pacientes con cáncer avanzado ${ }^{27}$.

\section{Estrategias para el manejo de síntomas (manejo} farmacológico y no farmacológico):

El tratamiento del delirio consiste básicamente en la sedación paliativa la cual podría realizarse con antipsicóticos típicos como el haloperidol y la clorpromazina, antipsicóticos atípicos como la olanzapina, la risperidona y la quetiapina o benzodiazepinas como el Lorazepam ${ }^{35}$. Aunque vale la pena contemplar en primera instancia la prevención y la detección temprana ${ }^{45}$, estas siguen siendo un desafío difícil en pacientes con cáncer terminal ${ }^{30}$.

Para el manejo del delirio es necesario tener en cuenta el estado de la enfermedad del paciente, la calidad de vida previa y los deseos expresados anteriormente con respecto de los objetivos de la atención. Coincidiendo con la afirmación de Rifa y Pons ${ }^{35}$, y, Sharon ${ }^{29}$, quienes consideran que los antipsicóticos son los agentes farmacoterapéuticos más comúnmente utilizados para el control sintomático aunque en casos con síntomas refractarios, puede ser necesaria una sedación paliativa. En este sentido Caraceni y Simonetti ${ }^{46}$, afirman que un enfoque interdisciplinario para el manejo y la investigación del delirio mejorarían la comprensión de un síndrome caracterizado por una gran carga de síntomas y una limitación pronóstica.

El papel de los cuidadores es necesario para mejorar la experiencia y para ayudar al paciente ${ }^{47}$ a manejar adecuadamente este síntoma ${ }^{48,49}$. Esta afirmación coincide con los resultados de Irwin, et al. ${ }^{26}$ en los cuales se menciona que, el empleo de intervenciones tanto farmacológicas como no farmacológicas, incluidas la educación del paciente y los cuidados informales, mejoran los síntomas y alivia la angustia del paciente y la familia, ya sea que el delirio sea reversible o irreversible, hiperactivo o hipoactivo. Todas las intervenciones se pueden proporcionar en cualquier entorno de atención, incluidos los hogares de los pacientes.

La agitación puede ser tratada como primera elección mediante la adaptación del medio, que puede ser respaldada por la prescripción de un medicamento antidepresivo no tricíclico sedante ${ }^{36}$, mediante el control de la deshidratación del paciente, el uso de

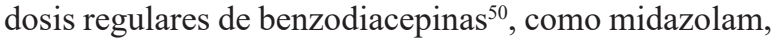
lorazepam, diazepam, clonazepam; y neurolépticos como haloperidol, tioridazina, clorpromazina, y risperidona ${ }^{7}$. Acompañada de terapias complementarias para reducir la ansiedad y la percepción del dolor, se considera necesario inducir la relajación y promover la comodidad y el bienestar en vista de que la agitación puede ser incluso una manifestación del delirio terminal ${ }^{35}$. En este sentido, autores como Boockvar, et al. ${ }^{51}$, mencionan el uso de terapias táctiles, a través del toque estructurado, como el uso de las energías del cuerpo para sanar usando las manos aunque estas no necesariamente hagan contacto físico con el paciente. Este tipo de técnicas puede aplicarse en cualquier entorno y es particularmente útil para pacientes al final de la vida.

Como estrategias no farmacológicas adicionales, $\mathrm{Akechi}^{31}$ menciona que, controlar los síntomas de agitación con una cuidadosa consideración de los deseos familiares ambivalentes, a quienes es necesario asistir y explicar el curso esperado con los cambios diarios, proporcionar información sobre la patología que ocasiona el delirio y respetar el mundo subjetivo del paciente, puede ser útil como estrategias de cuidado. Por otra parte, programas como el de almuerzo dos veces por semana llamado Lunch Bunch, también ha demostrado ser funcional en el manejo del delirio, pues proporcionan un medio para la estimulación mental y física que permite a los pacientes abrirse y hablar sobre los sentimientos ocultos de soledad y aislamiento, comenzando así un "viaje de sanación espiritual y emocional" ${ }^{52}$.

La confusión, así como otros tantos síntomas neurológicos, ha sido tratada en cuidados paliativos a través de la sedación paliativa ${ }^{53}$ y la hidratación del paciente, a pesar que estas prácticas sean bastante cuestionadas al contemplar el costo-beneficio, donde pueden mejorar el síntoma pero empeorar el edema cerebral, pulmonar y periférico ${ }^{54}$; por tanto los planes de búsqueda deben discutirse con el paciente y los familiares ${ }^{53}$.

Los enfoques farmacológicos para el control de las convulsiones en la atención al final de la vida son variables, a menudo excediendo las recomendaciones de la dosis ${ }^{55}$. Los fármacos más usados son el diazepam, el midazolam, el levetirazetam, la oxcarbazepina y el Valproato ${ }^{56}$. León Ruiz, et al. ${ }^{57}$, recomiendan usar fármacos antiepilépticos con presentación vía parenteral (preferiblemente intravenosa) y un perfil bajo de interacciones. Diazepam y/o midazolam serían los más idóneos para la fase aguda, y levetiracetam, ácido valproico y/o lacosamida para casos refractarios y/o como tratamiento crónico. Adicionalemente, como medida no farmacológica, se recomienda capacitar a 
los cuidadores de pacientes con necesidades paliativas en el reconocimiento y manejo temprano de las crisis prolongadas o repetitivas lo cual puede ayudar a reducir la ansiedad en el paciente, la angustia en la familia y evitar algunas visitas a urgencias ${ }^{56}$.

La espasticidad puede ser tratada con relajantes musculares de acción central como el baclofeno y las benzodiacepinas de acción prolongada como diazepam y clonazepam ${ }^{56}$. Se recomienda comenzar con una dosis baja y ajustarla gradualmente hasta lograr el efecto deseado ${ }^{58}$. El tratamiento no farmacológico de la espasticidad utiliza terapia física, posicionamiento y aparatos de ayuda y apoyo durables. Los estiramientos en pacientes con parálisis cerebral muestran que la elongación pasiva aumenta la extensión de los movimientos y reduce la espasticidad. Estas terapias pueden brindar beneficios significativos evitando los efectos secundarios y las interacciones típicas de los tratamientos farmacológicos ${ }^{56}$.

Las mioclonías deben prevenirse evitando la administración conjunta de antieméticos y es necesario que los profesionales de cuidados paliativos comiencen con un examen cuidadoso del síntoma para establecer el tratamiento adecuado. Debe explicarse a la familia cuales son las causas del síntoma y así evitar angustia; si no hay causa tratable el fármaco de elección es el midazolam, entre 10-30 mg en infusión continua vía subcutánea o IV para el caso de adultos ${ }^{35}$. En este sentido, se hace imperativo mencionar que, los cuidados paliativos para la enfermedad de Parkinson, en los que se presentan un número indeterminado de mioclonías (National Institute of Neurological Disorders and Stroke), son un área en rápido crecimiento que ofrece grandes posibilidades de mejorar el aprendizaje y el abordaje para los pacientes y sus cuidadores. Existe sin embargo la necesidad de investigación metodológica, observacional e intervencionista de abordar de manera exclusiva las necesidades únicas de individuos que padecen la Enfermedad de Parkinson ${ }^{45}$.

Se destacan los resultados de Romeo, et al..$^{59}$, quienes realizaron un estudio en el que pudo demostrar la efectividad de la acupuntura en la reducción y alivio de los síntomas que comúnmente afectan la calidad de vida del paciente, algunos de ellos con enfermedades neurológicas. La acupuntura redujo efectivamente los síntomas como la depresión y la ansiedad y mejoró las sensaciones de bienestar. Asimismo, Roberts y Campbell ${ }^{60}$, reflexionan sobre el potencial de las enfermeras de cuidados paliativos en un entorno de hospicio para apoyar a los pacientes con agitación y angustia al final de la vida mediante el uso de la técnica M, la cual se puede aprender en dos días, puede aplicarse en cualquier entorno y es particularmente útil para pacientes al final de la vida con dolor y tejido cutáneo frágil.

Por último, es importante considerar que, para determinar cómo se debe realizar la mejor atención en pacientes desde el ámbito individual, se requiere la síntesis de datos de la evaluación integral, incluidos los objetivos de atención y los valores del paciente, así como el conocimiento del estado de la enfermedad teniendo en cuenta la percepción del paciente y los enfoques basados en la evidencia ${ }^{61}$.

\section{Resultado del síntoma}

El cluster de los síntomas neurológicos hacen parte de los síndromes geriátricos más frecuentes en pacientes hospitalizados ${ }^{62}$ y se asocia con una cantidad significativa de morbilidad y mortalidad ${ }^{63}$, angustia tanto para el paciente como para su familia ${ }^{31}$, estancias hospitalarias prolongadas, deterioro funcional $\mathrm{y}$ disminución de la calidad de vida ${ }^{64}$. Se trata por tanto de una condición clínica exigente que complica la historia de los pacientes y las estrategias para la detección temprana, la prevención y el manejo aún son insuficientes. Se debe combinar una evaluación diagnóstica cuidadosa y una atención adecuada para satisfacer las necesidades de pacientes con diferentes perspectivas clínicas y carga de enfermedad ${ }^{46}$.

Coincidiendo con las afirmaciones anteriores, Young y Inouye ${ }^{65}$ aseveran que el delirio en un paciente hospitalizado con enfermedad avanzada se ha asociado con un doble aumento de la mortalidad, un incremento en los días de hospitalización y una pobre recuperación física y cognitiva que se relaciona con la necesidad de un mayor tiempo en atención institucional. Este síntoma persiste en aproximadamente un tercio de los pacientes $^{66}$ y su recurrencia representa un peor pronóstico en el impacto del síntoma para los pacientes, los cuales pueden experimentar recuerdos del evento que encuentran desagradables y perturbadores. Viloria Jiméne $z^{67}$ encontró que además del delirio, las alteraciones del sueño repercuten negativamente en la calidad de vida del paciente y su cuidador e incluso en la esfera socioeconómica debido a su elevada frecuencia en adultos mayores.

Por su parte, Cuervo Pinna, et al. ${ }^{44}$ ejecutaron una prueba de comparación de medidas emparejadas con el fin de observar la mejoría en el control sintomático, utilizando 
para ello el procedimiento de Kaplan Meyer para obtener así un análisis de supervivencia. Como resultado, se evidenció que hubo una mejoría estadísticamente significativa en los síntomas como depresión, ansiedad y bienestar después de una intervención del Equipo de Cuidados Paliativos y los pacientes refirieron percibir una mejor calidad de vida.

Las características específicas de las enfermedades neurológicas que afectan los cuidados paliativos y la calidad de vida de los pacientes fueron identificadas por. Gofton, et al. ${ }^{68}$ en un análisis cuantitativo: a) líneas de tiempo de progresión de la enfermedad, b) barreras a la comunicación derivadas de la enfermedad, c) variabilidad a lo largo de la progresión de la enfermedad y d) amenaza a la personalidad derivada de trastornos funcionales y cognitivos relacionados con la enfermedad neurológica. En cuanto a los desafíos que representan y complican el cuidado paliativo neurológico se mencionan: 1) incertidumbre con respecto al pronóstico, disponibilidad de apoyo y trayectoria de la enfermedad, 2) inconsistencia en información, actitudes y habilidades entre proveedores de atención, equipos de atención, cuidadores y familias, y 3) angustia existencial específica de la enfermedad neurológica, incluida la angustia emocional, psicológica y espiritual que resulta de la pérdida de la función, la autonomía y la muerte ${ }^{68}$.

Los pacientes alcanzan hitos clínicos como la dependencia de sillas de ruedas o las caídas frecuentes desde el principio de la enfermedad y pueden vivir con estas cargas acumuladas durante algunos años ${ }^{69}$. El reconocimiento de los beneficios de enfatizar sobre el efecto de condiciones no malignas y progresivas sobre la calidad de vida evidencia la necesidad de cuidados paliativos con enfoque integrativo. Trastornos como la atrofia multisistémica y la parálisis supranuclear progresiva por ejemplo, no solo conllevan una pesada carga de síntomas equivalente a la de los pacientes con cáncer avanzado, sino que los pacientes experimentan una morbilidad significativa durante un período prolongado de tiempo ${ }^{69}$.

\section{Discusión}

La educación ayuda a proporcionar cuidados paliativos de calidad $^{70}$, pues empodera a los profesionales de enfermería en el reconocimiento de desequilibrios en el bienestar, para actuar de manera rápida $\mathrm{y}$ efectiva y aliviar algunas situaciones desagradables en el paciente. Para la administración adecuada de la medicación de rescate, enfermería debe conocer los métodos de evaluación sintomática, farmacocinética y farmacodinámica de los medicamentos, los intervalos de tiempo que deben transcurrir entre los diferentes rescates ${ }^{71}$. El personal de enfermería ha de saber reconocer la descompensación, actuar rápida y eficazmente ${ }^{72} \mathrm{y}$ formular planes de cuidado anidados en taxonomías aceptadas a nivel mundial e incluidas en la NANDA (International Nursing Diagnoses: Definitions \& Classification $)^{73}$.

El contexto de los cuidados paliativos, permite estructurar sistemáticamente planes de cuidado, específicos para el manejo de los síntomas neurológicos, pues se identifican diagnósticos como: riesgo de lesión, conocimientos deficientes y deterioro de la interacción social. Tratar mediante intervenciones como: precauciones contra las convulsiones, identificación de riesgos, enseñanza: proceso de enfermedad, enseñanza: medicamentos prescritos, enseñanza: dieta prescrita, enseñanza: proceso de enfermedad, aumentar los sistemas de apoyo, y modificación de la conducta: habilidades sociales. Para llegar a obtener resultados como: control de las convulsiones, control del riesgo, ambiente seguro del hogar, conocimiento: cuidados en la enfermedad, habilidades de interacción social, y participación en actividades de ocio.

Finalmente la experiencia de los síntomas, de menor a mayor, hace que millones de pacientes visiten a sus proveedores de atención médica cada año. Los síntomas no solo crean angustia, sino que también interrumpen el funcionamiento social. Es por ello que el manejo de los síntomas y sus resultados a menudo se vuelven responsabilidad del profesional de salud reflejado en el paciente y los miembros de su familia ${ }^{74}$. Los proveedores de servicios de salud tienen dificultades para desarrollar estrategias de manejo de los síntomas que se pueden aplicar a través de los entornos agudos y de atención domiciliaria, ya que pocos modelos de gestión de los síntomas se han probado empíricamente. Hasta la fecha, la mayoría de las investigaciones sobre los síntomas se han dirigido hacia el estudio de un solo síntoma, como el dolor o la fatiga, o hacia la evaluación de los síntomas asociados. Es por ello que se deben aplicar enfoques que mejoren la comprensión de los síntomas, la gestión y la proporción de instrucciones que permitan seleccionar intervenciones clínicas, informar la investigación y unir una serie de síntomas asociados con una variedad de enfermedades y afecciones, para llegar a la integración de la ciencia desde los diferentes campos ${ }^{60}$. 


\section{Conclusiones}

El aporte multidisciplinario con frecuencia optimiza el control de los síntomas y la calidad de vida ${ }^{32}$ pero enfermería es responsable de detectar las respuestas humanas que son objeto de cuidado. Cuando el paciente se encuentra al final de la vida, los profesionales de enfermería tienen preocupaciones que por lo general incluyen distinguir entre el dolor y la agitación, equilibrar los riesgos de sub y sobremedicación y la posibilidad de acelerar la muerte ${ }^{75}$.

Así mismo es importante que el personal de enfermería se apropie de escalas de medición que permiten una adecuada evaluación y respuesta a los síntomas mediante el diagnóstico temprano para un posterior tratamiento y manejo, dentro de las escalas más usadas en el abordaje neurológico tenemos la monitorización del nivel de sedación según la escala de Ramsay ${ }^{64}$ y la Escala de Evaluación del Delirio como uno de los métodos de detección más importantes del mundo ${ }^{74}$.

El reconocimiento de los síntomas y su impacto en pacientes y sus cuidadores es un aspecto importante de enfermería, quienes se encuentran bien posicionados para realizar observaciones en el paciente ${ }^{36}$, documentar los cambios a lo largo del tiempo, implementar intervenciones farmacológicas y no farmacológicas ${ }^{76}$, educar y apoyar a los pacientes y sus familias. La búsqueda de las etiologías, el desarrollo de planes de atención individualizados con los objetivos del paciente y la aprobación del beneficio de la consulta/derivación se consideran funciones clave para los proveedores de cuidados paliativos de todas las disciplinas ${ }^{36}$.

Se necesitan enfoques innovadores para alinear los comportamientos profesionales de la salud con el mejor cuidado $^{77}$, donde se incluya una atención integral ${ }^{52}$, que mejore significativamente la atención al paciente.

\section{Referencias}

1. Who global atlas of palliative care. World Health Organization 2014.

2. Hirtz D, Thurman DJ, Gwinn-Hardy K, Mohamed M, Chaudhuri AR, Zalutsky R. How common are the common neurologic disorders? Neurology. 2007; 68(5): 326-337. doi: 10.1212/01. wnl.0000252807.38124.a3.

3. Mejía-Arango S, Miguel Jaimes A, Villa A, Ruiz L, Gutiérrez L. Deterioro cognitivo y factores asociados en adultos mayores en México. Salud Pública Mex. 2007; 49(4): 1-12.
4. Kurent JE. Palliative care in specific neurological diseases. Contin Lifelong Learn Neurol. 2005; 11: 33-77. doi: 10.1212/01.CON.0000293665.54027.2c.

5. Anderson GF, Chu E. Expanding priorities-confronting chronic disease in countries with low income. N Engl J Med. 2007; 356(3): 209-211. doi: 10.1056/NEJMp068182.

6. Corona T, Román GC. Multiple Sclerosis in Latin America. Neuroepidemiol. 2006; 26(1): 1-3. doi: 10.1159/000089230.

7. Pradilla G, León-Sarmiento FE. Esclerosis múltiple en Colombia: cerrando la brecha. Acta Neurol Colomb. 2007; 23(1): 3.

8. Fachado AA, Martínez NS, Roselló MM, Ria JJV, Oliver EB, García RG, et al. Spanish adaptation and validation of the supportive \& palliative care indicators tool - SPICT-ES ${ }^{\mathrm{TM}}$. Rev Saúde Pública. 2018; 52: 3. doi: 10.11606/s15188787.2018052000398.

9. Twycross R. Medicina paliativa: filosofía y consideraciones éticas. Acta Bioeth. 2000; 6(1): $27-$ 46. doi: 10.4067/S1726-569X2000000100003.

10. Gooding MP, Amaya E, Parra M, Ríos AM. Prevalencia de las demencias en el municipio de Neiva 2003-2005. Acta Neurol Colomb. 2006; 22(3): 243-248.

11. Silva FA, Zarruk JG, Quintero C, Arenas W, Rueda-Clausen CF, Silva SY, et al. Enfermedad cerebrovascular en Colombia. Rev Col Cardiol. 2006; 13(2): 85-89.

12. Toro J, Sarmiento OL, Díaz del Castillo A, Satizábal CL, Ramírez JD, Montenegro AC, et al. Prevalence of multiple sclerosis in Bogotá, Colombia. Neuroepidemiology. 2007; 28(1): 33-38.

13. Oliver DJ, Borasio GD, Caraceni A, de Visser M, Grisold W, Lorenzl S, et al. A consensus review on the development of palliative care for patients with chronic and progressive neurological disease. Eur J Neurol. 2016; 23(1): 30-38. doi: 10.1111/ ene. 12889 .

14. Carver AC, Foley KM. Symptom assessment and management. Neurol Clin. 2001; 19(4): 921-947.

15. León MX, Flórez SP, Torres M, Rengifo ML, Prada D. La importancia de evaluar síntomas $\mathrm{y}$ alteraciones funcionales en enfermedades neurológicas crónicas: experiencia en cuidado paliativo y rehabilitación en una institución colombiana. Aquichan. 2010; 10(3): 228-243.

16. Ferrell BR, Coyle N, Paice JA. Oxford Textbook of Palliative Nursing. 4ed. Oxford: Oxford University Press. 2015.

17. Dodd M, Janson S, Facione N, Faucett J, Froelicher ES, Humphreys J, et al. Advancing the science of 
symptom management. J Adv Nurs. 2001; 33(5): 668-676.

18. Santos CM, Pimenta CA, Nobre MRC. The PICO strategy for the research question construction and evidence search. Rev Lat Am Enfermagem. 2007; 15(3): 508-511.

19. Moher D, Liberati A, Tetzlaff J, Altman DG, PRISMA Group. Preferred reporting items for systematic reviews and meta-analyses: the PRISMA statement. PLoS Med. 2009; 6(7): e1000097. doi: 10.1371/journal.pmed.1000097.

20. Cwiekala K, Parkyn B, Modliszewska K, Analysis of University of California in San Francisco (UCSF) Symptom Management theory and theory Implication for Persons with Neurological Disorders/Diseases. J Neurol Neurosurg Nursing. 2017; 6(2): 55-65. doi: 10.15225/PNN.2017.6.2.2.

21. Kerr CW, Donnelly JP, Wright ST, Luczkiewicz DL, McKenzie KJ, Hang PC, et al. Progression of delirium in advanced illness: a multivariate model of caregiver and clinician perspectives. J Palliat Med. 2013;16(7): 768-773. doi: 10.1089/jpm.2012.0561.

22. Mystakidou K, Parpa E, Katsouda E, Galanos A, Vlahos L. Pain and desire for hastened death in terminally ill cancer patients. Cancer Nurs. 2005; 28(4): 318-324.

23. Spoozak L, Seow H, Liu Y, Wright J, Barbera L. Performance status and symptom scores of women with gynecologic cancer at the end of life. Int J Gynecol Cancer. 2013; 23(5): 971-978. doi: 10.1097/IGC.0b013e318291e5ef.

24. Warth M, Kessler J, Bardenheuer HJ. Insomnia and autonomic function predict well-being in patients receiving palliative care. J Palliat Med. 2017; 20(12): 1395-1399. doi: 10.1089/jpm.2017.0032.

25. Covarrubias A, Hernández EE, Ruiz S, López C-Estrada M. Assessment of pain and other symptoms in Mexican patients with advanced illness. J Pain Palliat Care Pharmacother. 2014; 28(4): 394398. doi: 10.3109/15360288.2014.959235.

26. Irwin SA, Pirrello RD, Hirst JM, Buckholz GT, Ferris FD. Clarifying delirium management: practical, evidenced-based, expert recommendations for clinical practice. J Palliat Med. 2013;16(4): 423435. doi: 10.1089/jpm.2012.0319.

27. Morita T, Chinone Y, Ikenaga M, Miyoshi M, Nakaho T, Nishitateno K, et al. efficacy and safety of palliative sedation therapy: a multicenter, prospective, observational study conducted on specialized palliative care units in Japan. J Pain Symptom Manage. 2005; 30(4): 320-328.

28. Rubio ML, Adalid C, de Castro C. Dilemas éticos en el cuidado de un paciente terminal: una visión desde la asistencia primaria. Aten Primaria. 2001; 27: 621-622.

29. Sharon K. Delirium in older persons. N. Engl J Med. 2006; 354: 1157-1165. doi: 10.1056/ NEJMra052321.

30. Gagnon P, Allard P, Gagnon B, Mérette C, Tardif F. Delirium prevention in terminal cancer: assessment of a multicomponent intervention. Psychooncology. 2012; 21(2): 187-194. doi: 10.1002/pon.1881.

31. Akechi T. Desire for early death in cancer patients and clinical oncology. Jpn J Clin Oncol. 1999; 29(12): 646-646. doi: https://doi.org/10.1093/ jjco/29.12.646.

32. Alt-Epping B, Stäritz AE, Simon ST, Altfelder N, Hotz T, Lindena $G$, et al. What is special about patients with lung cancer and pulmonary metastases in palliative care? Results from a nationwide survey. J Palliat Med. 2012; 15(9): 971-977. doi: 10.1089/ jpm.2011.0434.

33. Öhlén J, Russell L, Håkanson C, Alvariza A, Fürst $\mathrm{CJ}$, Årestedt $\mathrm{K}$, et al. Variations in care quality outcomes of dying people: latent class analysis of an adult national register population. J Pain Symptom Manage. 2017; 53(1): 13-24. doi: 10.1016/j. jpainsymman.2016.08.006.

34. Benítez M, Cabrejas A, Fernández R, Pérez M. Complicaciones psiquiátricas, neurológicas $\mathrm{y}$ cutáneas en el paciente con enfermedad en fase terminal. Aten Primaria 2002; 30 (3): 179-182.

35. Rifà F, Pons S. El final de la vida. Síntomas y complicaciones más frecuentes. Servicio de Oncología Médica. Hospital Universitario Son Dureta. Palma de Mallorca (Illes Balears). Manual SEOM de Cuidados Continuos.

36. Hosie A, Agar M, Lobb E, Davidson PM, Phillips J. Palliative care nurses' recognition and assessment of patients with delirium symptoms: a qualitative study using critical incident technique. Int J Nurs Stud. 2014; 51(10):1353-1365. doi: 10.1016/j. ijnurstu.2014.02.005.

37. Verstraete L, Joosten E, Milisen K. Opinions of physicians and nurses regarding the prevention, diagnosis and management of delirium. Tijdschr Gerontol Geriatr. 2008; 39: 26-34.

38. Ben-Zacharia AB, Lublin FD. Palliative care in patients with multiple sclerosis. Neurol Clin. 2001; 19(4): 801-827.

39. Hildreth KL, Church S. Evaluation and management of the elderly patient presenting with cognitive complaints. Med Clin North Am. 2015; 99(2): 311335. doi: 10.1016/j.mcna.2014.11.006.

40. Tuffrey-Wijne I. The palliative care needs of 
people with intellectual disabilities: a literature review. Palliat Med. 2003; 17(1): 55-62. doi: 10.1191/0269216303pm668oa.

41. Heckel M, Stiel S, Ostgathe C. Smell and taste in palliative care: a systematic analysis of literature. Eur Arch Oto-Rhino-Laryngol. 2015; 272(2): 279288. doi: 10.1007/s00405-014-3016-4.

42. Fischbeck S, Maier B-O, Reinholz U, Nehring C, Schwab R, Beutel ME, et al. Assessing somatic, psychosocial, and spiritual distress of patients with advanced cancer: development of the Advanced Cancer Patients' Distress Scale. Am J Hosp Palliat Care. 2013; 30(4): 339-346. doi: $10.1177 / 1049909112453640$.

43. Kutner JS, Blatchford PJ, Taylor DH, Ritchie CS, Bull JH, Fairclough DL, et al. Safety and benefit of discontinuing statin therapy in the setting of advanced, life-limiting illness: a randomized clinical trial. JAMA Intern Med. 2015; 175(5): 691700. doi: 10.1001/jamainternmed.2015.0289.

44. Pinna C, Redondo M, Sánchez Correas MA, Ramos Jiméneza MA, Mota Vargas R. Control of symptoms by a palliative care team. Preliminary results. Med Paliativa. 2010; 17: 96-102.

45. Benze G, Alt-Epping B, Nauck F. Palliative care in oncology | Bernd Alt-Epping | Springer book/9783662462010.2015.

46. Caraceni A, Simonetti F. Palliating delirium in patients with cancer. Lancet Oncol. 2009; 10(2): 164-172. doi: 10.1016/S1470-2045(09)70018-X.

47. Sternjswärd J, Foley K, Ferris F. The Public Health Strategy for Palliative Care. J Pain Symptom Manage 2007 ; 33( 5): 486-493.

48. Merel SE, Merel S, DeMers S, Vig E. Palliative care in advanced dementia. Clin Geriatr Med. 2014; 30(3): 469-492. doi: 10.1016/j.cger.2014.04.004

49. Abrahm JL. Advances in palliative medicine and end-of-life care. Annual Review of Medicine. 2011; 62: 187-199. https://doi.org/10.1146/annurevmed-050509-163946.

50. Fuentes C, Schonfelldt A, Rojas O, Briganti M, Droguettd M, Muñoz E, et al. Delirium en el paciente Oncológico. Rev Medica clínica Condes. 2017; 28: 855-865. doi: 10.1016/j.rmclc.2017.10.009.

51. Boockvar KS, Teresi JA, Inouye SK. Preliminary data: an adapted hospital elder life program to prevent delirium and reduce complications of acute illness in long term care delivered by certified nursing assistants. J Am Geriatr Soc. 2016; 64(5): 1108-1113. doi: 10.1111/jgs.14091.

52. Nedjat-Haiem FR, Carrion IV, Gonzalez K, Ell K, Thompson B, Mishra SI. Exploring health care providers' views about initiating end-of-life care communication. Am J Hosp Palliat Care. 2017; 34(4): 308-317. doi: 10.1177/1049909115627773.

53. Amblàs-Novellas J, Murray SA, Espaulella J, Martori JC, Oller R, Martinez-Muñoz M, et al. Identifying patients with advanced chronic conditions for a progressive palliative care approach: a crosssectional study of prognostic indicators related to end-of-life trajectories. BMJ Open. 2016; 6(9): e012340. doi: 10.1136/bmjopen-2016-012340.

54. Wusthoff CJ, Shellhaas RA, Licht DJ. Management of common neurologic symptoms in pediatric palliative care: seizures, agitation, and spasticity. Pediatr Clin North Am. 2007; 54(5): 709-733. doi: 10.1016/j.pcl.2007.06.004.

55. LeGrand SB. Delirium in palliative medicine: a review. J Pain Symptom Manage. 2012; 44(4): 583594. doi: 10.1016/j.jpainsymman.2011.10.013.

56. Villegas JA, Antuña MJ. Cuidados Paliativos Pediátricos. Bol Pediatr. 2012; 52: 131-145.

57. León M, Rodríguez ML, Sanjuán L, Pérez MT, Ibáñez F, Arce Arce S, et al. Guía para el manejo de las crisis epilépticas en cuidados paliativos: propuesta de un modelo actualizado de práctica clínica basado en una revisión sistemática de la literatura. Neurología. 2017. doi: https://doi. org/10.1016/j.nrl.2016.11.010.

58. Chafic Y, Karam, Paganoni S, Joyce N, Carter GT, Bedlack R. Palliative Care Issues in Amyotrophic lateral sclerosis: an evidenced-based review. Am J Hosp Palliat Care. 2016; 33(1): 84-92. doi: 10.1177/1049909114548719.

59. Romeo MJ, Parton B, Russo RA, Hays LS, Conboy L. Acupuncture to treat the symptoms of patients in a palliative care setting. Explore NY. 2015;11(5): 357-362. doi: 10.1016/j.explore.2015.06.001.

60. Roberts K, Campbell H. Using the $M$ technique as therapy for patients at the end of life: two case studies. Int J Palliat Nurs. 2011;17(3): 114-118. doi: 10.12968/ijpn.2011.17.3.114.

61. Lynch M, Dahlin C, Bakitas M. Bowel obstruction and delirium: managing difficult symptoms at the end of life. Clin J Oncol Nurs. 2012;16(4): 391-398. doi: 10.1188/12.CJON.391-398.

62. Perelló C. Valoración del riesgo de delirium en pacientes mayores hospitalizados. Rev Esp Geriatr Gerontol. 2010; 45(5): 285-290. doi: 10.1016/j. regg.2010.03.011.

63. Chun-Kai F, Hong-Wen Ch. Prevalencia, detección y tratamiento del delirio en pacientes con cáncer terminal: una encuesta prospectiva. Jpn J Clin Oncol. 2008; 38(1): 56-63 doi: 10.1093/jjco/hym155.

64. Elsayem AF, Elzubeir H, Brock P, Todd K. Integrating palliative care in oncologic emergency 
departments: Challenges and opportunities. World J Clin Oncol. 2016; 7(2): 227-233. doi:10.5306/wjco. v7.i2.227.

65. Young J, Inouye SK. Delirium in older people. BMJ. 2007; 334(7598): 842-846. doi: 10.1136/ bmj.39169.706574.AD.

66. Siddiqi N, House AO, Holmes JD. Occurrence and outcome of delirium in medical in-patients; a systematic literature review. Age Ageing. 2006; 35 : 350-364. doi: 10.1093/ageing/afl005.

67. Viloria Jiménez MA. Enfermedades neurológicas y psiquiátricas en el anciano. Med - Prog Form Médica Contin Acreditado. 2014;11(62): 3659-3676.

68. Gofton TE, Chum M, Schulz V, Gofton BT, Sarpal A, Watling C. Challenges facing palliative neurology practice: a qualitative analysis. J Neurol Sci. 2018; 385: 225-231. doi: 10.1016/j.jns.2017.12.008.

69. Wiblin L, Lee M, Burn D. Palliative care and its emerging role in multiple system atrophy and progressive supranuclear palsy. Parkinsonism Relat Disord. 2017; 34: 7-14. doi: 10.1016/j. parkreldis.2016.10.013.

70. Mailly M, Bénézech J-P. Observational study of delirium in palliative care -j.revinf.2016; 66(228):37-38] DOI: 10.1016/j.revinf.2016.12.011.

71. Dominguez Alvaréz R, Calderón Carrasco J, García Colchero F, Postigo Mota S, Alburquerque Medina E. Pharmacological treatment in palliative care. drug administration route, continuous subcutaneous infusion, adverse side effects, symptom management. Rev Enferm. 2015; 38(1): 54-58, 61-65.

72. Wettstein A. Basics of gerontopharmaceutic therapy and the therapy of agitation in old age. Praxis. 2009; 98(21): 1211-1217. doi: 10.1024/16618157.98.21.1211.

73. Herdman H. Clasificación completa de diagnósticos enfermeros NANDA-I 2015-2017. Barcelona: Elsevier; 2015.

74. Hardy K, Brown M. Delirium: a diagnostic dilemma. Part 1. Br J Nurs. 2015; 24(16): S9-14. doi: 10.12968/bjon.2015.24.Sup16.S9.

75. Spineli V, Kurashima AY, Gutiérrez M. The process of palliative sedation as viewed by physicians and nurses working in palliative care in Brazil. Palliat Support Care. 2015; 13(5): 1293-1299. doi: $10.1017 /$ S1478951514001278.

76. Grassi L, Caraceni A, Beltrami E, Borreani C, Zamorani M, Maltoni M, et al. Assessing delirium in cancer patients: the Italian versions of the Delirium Rating Scale and the Memorial Delirium Assessment Scale. J Pain Symptom Manage. 2001; 21(1): 59-68.
77. Heick A. Terminal phase hydration, pain and delirium. Ugeskr Laeger. 2009; 171: 2738-2741. 